\title{
Severe mycobacterium bovis BCG infection in an infant with partial dominant interferon gamma receptor deficiency-a case report
}

\begin{abstract}
Mendalian susceptibity to mycobacterial disease (MSMD) is a condition caused by selective susceptibility to weakly virulent bacteria in otherwise healthy patients without additional immunological abnormalities. It is an inherited, genetic disorder with variety of clinical presentation. Diagnosis is mandatory because the illness may get precipitated by BCG and other live vaccines. Estimating interleukin in serum can be considered as a diagnostic test. Immunological analysis is mandatory for confirming the diagnosis. Mutation analysis can be done to confirm the mutation and hence, prevent the disease in the next sibling by testing in utero. This condition can be treated with ATT as the first line treatment. If ineffective, can be given other modalities of treatment described. But relapses are common. Stem cell transplantation is the definitive treatment. We describe an infant diagnosed as partial dominant interferon gamma receptor deficiency (IFNGR1) deficiency, who responded to ATT.
\end{abstract}

Keywords: interferon gamma receptor, interleukins, anti tubercular therapy, mycobacteria
Volume 7 Issue 4 - 202 I

\author{
Jagruthi Reddy,' Priya Jose,' Mathew \\ Varghese, ${ }^{2}$ Peter Prasanth Kumar Kommu,' \\ K H Kishku ${ }^{2}$ \\ 'Department of Paediatrics, Pondicherry institute of Medical \\ Sciences, India \\ ${ }^{2}$ Department of Pulmonary medicine, Pondicherry Institute of \\ Medical Sciences, India
}

Correspondence: Priya Jose, Associate Professor, Department of Paediatrics, Pondicherry institute of Medical Sciences, Puducherry,Tel 9600635906, Email drjose.priya@gmail.com

Received: July II, 2021 | Published: August 13, 202 I
Abbreviations: ATT, anti-tubercular treatment; IL, interleukin; MSMD, mendalian susceptibity to mycobacterial disease

\section{Introduction}

Mendelian susceptibility to mitochondrial disease (MSMD) is a rare inherited condition, caused by one or more weakly virulent mycobacteria like BCG vaccination and non-tuberculous environmental mycobacteria. ${ }^{1}$ It is a disease that generally begins in childhood, with variety of manifestations like localized to disseminated life threatening infections. ${ }^{2}$ The etiology was first described in 1996. Since then 11 MSMD causing genes have been identified so far, and are manifested as 21 genetic disorders. ${ }^{3}$ It could be either because of impaired production or impaired response to Interferon (IFN) gamma. Disseminated tuberculous mycobacterial infection should be considered as a differential diagnosis for MSMD, but disseminated tuberculous mycobacterial infection is more common in immunosuppressive children. Even though most of the cases respond to Anti-tubercular treatment (ATT), additional IFN gamma may be needed in some cases. Hematopoietic stem cell transplantation can also be considered. ${ }^{2}$ This disease entity is hardly diagnosed and reported, might be because of lack of awareness about the progress of this genetic condition. Hence, we are reporting a case of BCG lymphadenitis with pulmonary involvement, that we have diagnosed for the first time in this tertiary care hospital, Puducherry.

\section{Case report}

3 months old girl who had a smooth transition perinatally, immunized and developed age appropriate milestones was brought by parents with history of fever and nasal discharge of 2 weeks duration. Fever subsided after 5 days. There was cough for 4-5 days, and increased work of breathing for 1 day. No history of poor feeding, no weight loss or lethargy. At 2 months of age, she was diagnosed with left axillary lymphadenitis for which she was treated with antibiotics.
Incision \& drainage was also done. There was no history of contact with tuberculosis.

On examination, she was pale, in respiratory distress, and tachypneic with bilateral subcostal and intercostal retractions. Saturation was $94 \%$ in room air. Left axillary lymph node of $2 \times 2 \mathrm{~cm}$, tender, warm, mildly erythematous was present. Healed scar was present over the lymph node. Anthropometric measurements were normal. Dry scaly lesions suggestive of ichthyosis vulgaris was present over the lower limbs. Chest examination revealed normal vesicular breath sounds with bilateral coarse crepitations. There was hepatosplenomegaly. Ejection systolic murmur was heard in the pulmonary area. Liver span was of $8 \mathrm{~cm}$, and spleen was palpated 4 $\mathrm{cm}$ below the left costal margin.

A diagnosis of bronchopneumonia was made. The distress was not improving with oxygen through nasal prongs, so the child was connected to hot humidified high flow nasal canula (HHHFNC). Chest $\mathrm{X}$ ray revealed non-homogenous opacity bilaterally (Figure 1) and hence IV broad spectrum antibiotics were given for 10 days. As the distress settled, HHHFNC was tapered, stopped and shifted to ward. The child developed high grade fever and worsening of symptoms with increased work of breathing in another 2 days, hence oxygen was restarted.

In view of pneumonia, anemia, hepatomegaly, splenomegaly, icthyosis vulgaris and axillary lymphadenitis, disseminated tuberculosis was suspected and worked up. Mantoux test showed induration with size of $20 \mathrm{~mm}$ (Figure 2), ESR was $124 \mathrm{~mm} / \mathrm{hr}$. Gene Xpert of nasopharyngeal aspirate was negative, AFB staining of nasopharyngeal aspirate was negative. Fine needle aspiration cytology from the lymph node revealed positive AFB on staining (Figure 3). Considering possibility of disseminated tuberculosis, the child was started on ATT. The symptoms were settling for 1-2 days and then recurring, with high total leucocyte count and very high CRP for the first one month of ATT. 


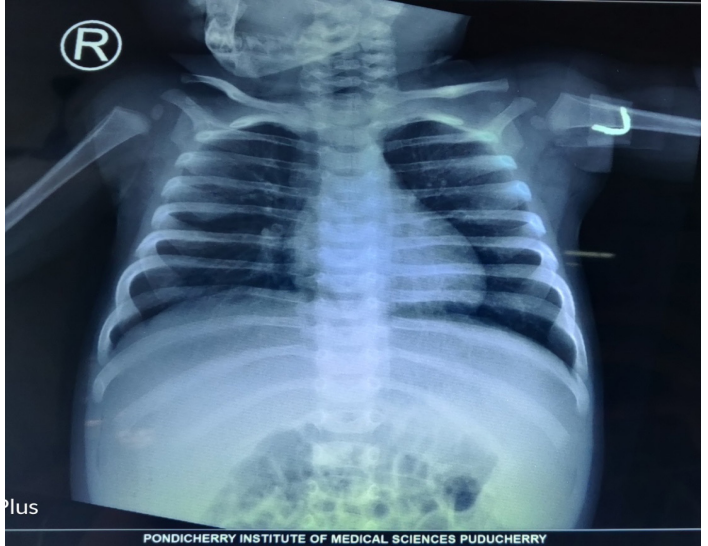

Figure I Chest $X$ ray of the patient at admission, showing non homogenous opacity bilaterally more on right side.

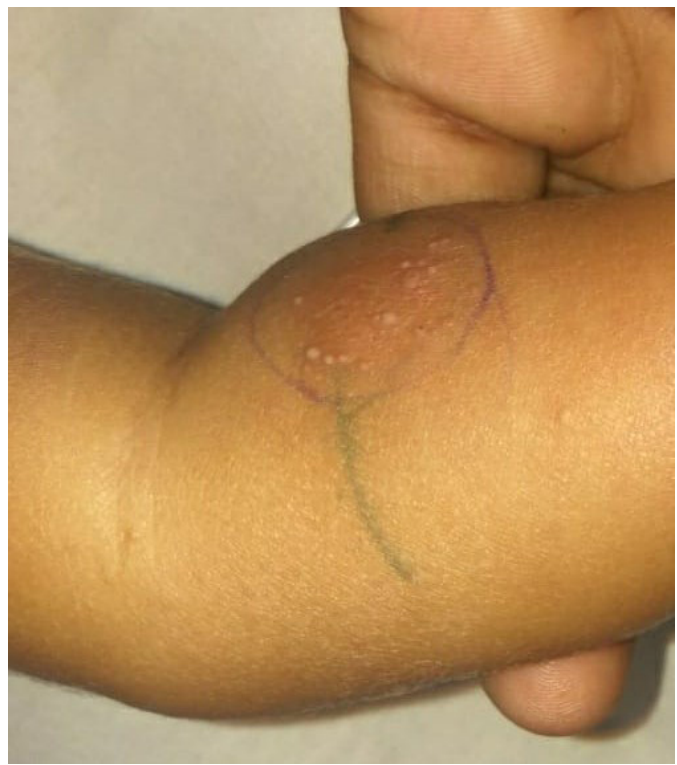

Figure 2 Strongly positive mantaux test of this baby.

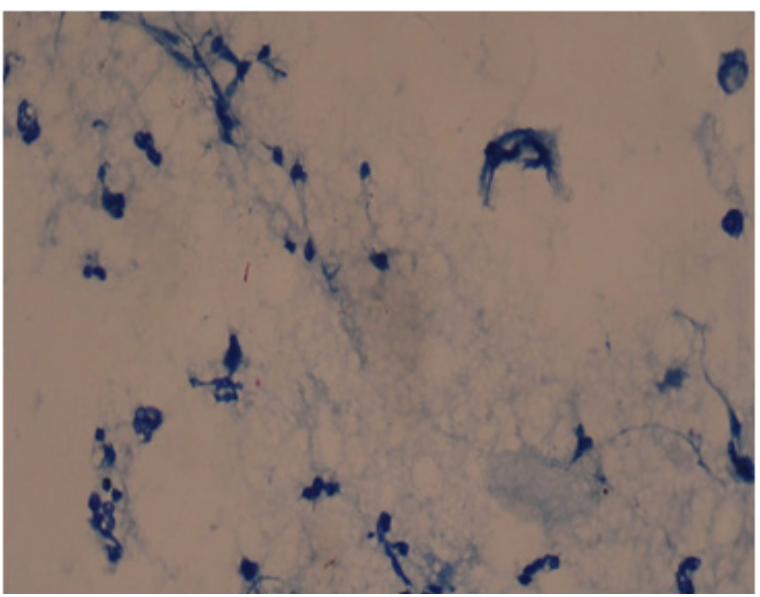

Figure 3 Demonstration of AFB in FNAC of lymph node.

ANA and Tandem mass spectroscopy were negative. No abnormal leucocytes/precursors found in peripheral smear. Antenatal records of mother rwas retroviral negative and was not done for the baby. Mendalian susceptibity to mycobacterial tuberculosis was suspected and the blood sample was sent for immunological analysis, revealed partial dominant IFNGR (Interferon gamma receptor)1 deficiency. So, ATT was continued for 6 months. The baby is asymptomatic and thriving well after 6 months of ATT.

\section{Discussion}

Mendalian susceptibity to mycobacterial tuberculosis (MSMD) is a condition caused by selective susceptibility to weakly virulent bacteria in otherwise healthy patients without additional immunological abnormalities. ${ }^{3}$ Disseminated mycobacterial infection can be associated with autoantibodies to IFN gamma as an acquired disease. The disease onset is usually during late childhood in partial form and presents as tuberculosis, salmonellosis, histiocytosis etc. ${ }^{2}$ Functional studies of Interferon gamma axis or Interleukin (IL)12 genotyping are the gold standard for the diagnosis of genetic defects. ${ }^{4}$

BCG vaccine is contraindicated when there is immunodeficiency. According to a study done by Toida and Nakta , 39 complicated cases with BCG vaccine were noted since 1951.Out of that,13 were found to have some form of primary immunodeficiency diseases when evaluated. Of those 13, 4 had IFNGR1 deficiency. ${ }^{5}$ Hoshina et al had analyzed the genetic background of patients diagnosed as MSMD over a period of 10 years and found out that 6 had mutation in IFNGR1. Among them, 4 patients had spontaneous occurrence similar to our case. ${ }^{6}$ The most common form is dominant partial IFNGR1 deficiency, which is a milder disease and has a good treatment response. Severe form is complete IFNGR1 deficiency. ${ }^{1}$

Novel methods like Whole exome sequencing and whole genome will help in further discovering new genetic disorders of MSMD. Usually patients with partial dominant IFNGR1 deficiency responds well to ATT. If there is no response, IFN gamma therapy can be given additionally. ${ }^{8}$ Mycobacterium bovis is resistant to pyrizanamide because of the expression of the pyrizinamidinase. There are reports available to describe the better efficacy of ATT if streptomycin can be added along with the first line in that kind of cases. ${ }^{9}$ It is more effective when Clarithromycin is added in suspicious cases of nontuberculous mycobacterium.

Treatment with second line drugs like fluroquinolones along with rifampicin and ethambuol (First line ATT) are more effective than first line treatment alone against multidrug resistant mycobacterium. ${ }^{10}$ High dose interferon gamma and levofloxacin are the most effective treatment in case the patient is intolerable to first line ATT. ${ }^{9}$ Full remission is difficult, relapses occur quite often after the discontinuation of ATT. BCG and other live vaccines are contraindicated, because it may precipitate the disease. ${ }^{8}$ Bone marrow transplantation is the only modality if all this medical management becomes ineffective. ${ }^{8}$

\section{Conclusion}

IFN receptor deficiency is a rare disorder, but to be considered in cases of BCG lymphadenitis. This type of partial immunodeficiency cases should be treated initially with conventional antituberculosis drugs like in our scenario. Then if resistant, other modalities of treatment can be tried.

\section{Acknowledgments}

We extend our gratitude to data collectors and study subjects.

\section{Conflicts of interest}

We have no conflict of interest in this article. 


\section{Funding}

None.

\section{References}

1. Darleguy A, Bost-Bru C, Pagnier A, et al. Mendelian susceptibility to mycobacterial disease: a case report of disseminated infection due to Mycobacterium avium. Arch Pediatr Organe Off Soc Francaise Pediatr. 2013;20(7):758-761.

2. Sarrafzadeh SA, Mahloojirad M, Nourizadeh M, et al. Mendelian Susceptibility to Mycobacterial Disease due to IL-12R $\beta 1$ Deficiency in Three Iranian Children. Iran J Public Health. 2016;45(3):370-375.

3. Rosain J, Kong X-F, Martinez-Barricarte R, et al. Mendelian susceptibility to mycobacterial disease: 2014-2018 update. Immunol Cell Biol. 2019;97(4):360-367.

4. Esteve-Solé A, Sologuren I, Martínez-Saavedra MT, et al. Laboratory evaluation of the IFN- $\gamma$ circuit for the molecular diagnosis of Mendelian susceptibility to mycobacterial disease. Crit Rev Clin Lab Sci. 2018;55(3):184-204.
5. Toida I, Nakata S. Severe adverse reactions after vaccination with Japanese BCG vaccine: a review. Kekkaku. 2007;82(11):809-824.

6. Hoshina T, Takada H, Sasaki-Mihara Y, et al. Clinical and host genetic characteristics of Mendelian susceptibility to mycobacterial diseases in Japan. J Clin Immunol. 2011;31(3):309-314.

7. Bustamante J, Boisson-Dupuis S, Abel L, et al. Mendelian susceptibility to mycobacterial disease: genetic, immunological, and clinical features of inborn errors of IFN- $\gamma$ immunity. Semin Immunol. 2014;26(6):454-470.

8. Remus N, Reichenbach J, Picard C, et al. Impaired interferon gammamediated immunity and susceptibility to mycobacterial infection in childhood. Pediatr Res. 2001;50(1):8-13.

9. Obinata $\mathrm{K}$, Lee $\mathrm{T}$, Niizuma $\mathrm{T}$, et al. Two cases of partial dominant interferon- $\gamma$ receptor 1 deficiency that presented with different clinical courses of bacille Calmette-Guérin multiple osteomyelitis. J Infect Chemother Off J Jpn Soc Chemother. 2013;19(4):757-760.

10. Fennelly GJ. Mycobacterium bovis versus Mycobacterium tuberculosis as a cause of acute cervical lymphadenitis without pulmonary disease. Pediatr Infect Dis J. 2004;23(6):590-591. 